\title{
Synthesis of melamine-formaldehyde-resin microspheres hybridized with aluminum-hydroxide
}

\author{
Junfu Zhu ${ }^{1, a}$, Shuzhen Wang ${ }^{2, b}$, and Zhixian $\mathrm{Hao}^{3, \mathrm{c}}$ * \\ ${ }^{1}$ Department of Chemistry, Tongji University, Siping Road 1239, Shanghai 200092, China \\ azhujunfu0@126.com, 'haozhixian@tongji.edu.cn
}

Keywords: Melamine-formaldehyde resin, alumina, core-shell

Abstract. A facile approach was introduced for the synthesis of hybrid-microspheres (MSs) of melamine formaldehyde resin (MF) and aluminum hydroxide ( $\mathrm{Al}$ ). Wherein, the uniform MF MSs were firstly prepared under acid conditions, and then hybridized with aluminum hydroxide under urea solution presence and decomposition conditions. These MS hybrids presented a core-shell structure, an average diameter of $3.2 \mu \mathrm{m}$ as same as that of MF MSs, 35\% content of the inorganic alumina and a high decomposition temperature high until to $750{ }^{\circ} \mathrm{C}$.

\section{Introduction}

MF is useful in some of important industrial areas, such as artificial board, tanning industry, adhesive, etc. [1,2]. Indeed, MF surface characteristics are variable even with their synthesis reaction conditions, analogous to that observed in the urea and formaldehyde resin [3,4]. In addition, the presence of numerous hydrophilic groups on the surface makes the MF capable of adsorbing inorganic ions under aqueous solution conditions that provide some new chances for us to further modify these useful materials. Transferring it into uniform microspheres is an important practice [5] in MF synthesis. Herein, we would like to modify MF MSs by aid of the infinite to inorganic aluminum ions and then transcribe them overall into MF-aluminum hydroxide (Al) micro-spherical hybrids under urea decomposition condition, which were expected to present the mixture characters of MF and aluminum hydroxide as well as a much more stability. This paper first presented these MF-Al MSs with a highly stability and 35\% alumina in, enabling it possible for us to design some new applications in environment treatment and detection.

\section{Experimental section}

Materials. Melamine, aqueous formaldehyde solution (37\%), acetic acid, urea ( $\geq 99.0 \%)$ and anhydrous ethanol $(\geq 99.7 \%)$ were purchased from Sinopharm Chemical Reagent Co., Ltd, and aluminium nitrate $(\geq 99.0 \%)$ from Shanghai ZhenXin Reagent Co., Ltd. All chemicals were used as received without any further purification.

Sample preparation and characterization. The melamine formaldehyde resin (MF) microspheres (MSs) were prepared following a standard literature procedure [6,7]. For a typical synthesis, $8.9 \mathrm{~mL}$ formaldehyde and $2.5 \mathrm{~g}$ melamine were sequentially added into $90 \mathrm{~mL}$ deionized water at $\mathrm{pH} 4.5$ calibrated with acetic acid, the mixture was then heated to $65{ }^{\circ} \mathrm{C}$ and maintained until it became a clear solution. After $30 \mathrm{~min}$ time of reaction at $65^{\circ} \mathrm{C}$ starting from a cloud state, the suspension was allowed to cool down to room temperature, the supernatant was removed via centrifugation and the 
MF MSs washed with water and ethanol for 3 times, respectively. The hybridization of aluminium-MF microspheres was performed at $85{ }^{\circ} \mathrm{C}$ for $3 \mathrm{~h}$ with $0.20 \mathrm{~g}$ MF MSs that were suspended in $40 \mathrm{~mL}$ deionized water containing $0.0375 \mathrm{~g}$ aluminium nitrate and $3.0 \mathrm{~g}$ urea. Following a cooling down and centrifugation step, the hybridized MSs collected were dried at $60{ }^{\circ} \mathrm{C}$ for $12 \mathrm{~h}$ and finally calcinated at $800{ }^{\circ} \mathrm{C}$ for $1 \mathrm{~h}$ to gain hollow alumina ones.

Scanning electron microscopy (SEM) was performed on Philips XL-30 at an accelerating voltage of $20 \mathrm{kV}$. Powder X-ray diffraction (XRD) was measured on a D8 advance diffraction meter (Bruker, Germany) with $\mathrm{Cu}-\mathrm{K} \alpha$ radiation at $40 \mathrm{kV}$ and $40 \mathrm{~mA}$, while a scanning rate of $2 \% \mathrm{~min}$ was adopted over a Bragg's angle range of $10-70^{\circ}(2 \theta)$. Fourier transform infrared spectra (FT-IR) were collected on a Thermo Nicolet NEXUS spectrometer at $4.00 \mathrm{~cm}^{-1}$ over 32 scans using $\mathrm{Pb}(\mathrm{SCN})_{2}$ as the internal standard presenting a characteristic peak at $2070 \mathrm{~cm}^{-1}$. The samples were sequentially mixed accurately with the same amount of the $\mathrm{Pb}(\mathrm{SCN})_{2}$ standard and pressed into $\mathrm{KBr}$ pellets after the grinding. Differential Thermal Analysis (DTA) of the samples was performed on a TA Instrument SDTQ-600 at a nitrogen flow rate of $18 \mathrm{~mL} \cdot \mathrm{min}^{-1}$.

\section{Results and Discussion}

A simple approach for the synthesis of MF-Al MSs. A schematic displayed in Fig. 1 was to simply illustrate our procedure for the synthesis of MF-Al MSs and to present what their composition will be. The uniform dispersed MF MSs were firstly prepared following the standard literature procedures and then hybridized in an aqueous solution containing aluminum nitrate and urea at $65{ }^{\circ} \mathrm{C}$ following a dispersion step under ultrasonic condition. The aluminum hydroxide can finally yield and deposit on the surface of the MF MSs due to its fine affinity to them, as shown in step (b). The step (c) demonstrates that these MF-Al MSs indeed posses a core-shell structure speculated from the shell structure after their calibration at $800^{\circ} \mathrm{C}$.

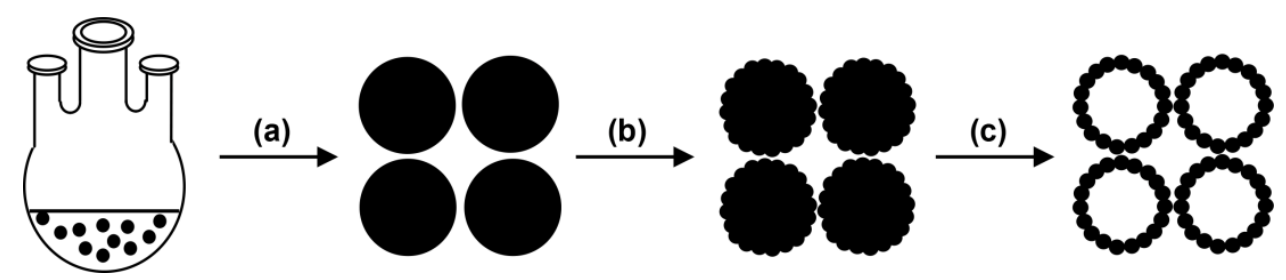

Fig. 1 Schematic for the synthesis of the MF-Al hybrids and the verification of their composition

(a) Synthesis of MF MSs followed by the filtration; (b) Synthesis of MF-Al hybrids via adsorption and deposition of alumina on MSs followed by their filtration and drying; (c) Calcination of the hybrids at $800{ }^{\circ} \mathrm{C}$ for alumina MSs.

Characterizations of MF-Al MSs. The XRD spectrum of the MF-Al MSs was presented in Fig. 2b with a broaden diffraction peak around $2 \theta=22.5^{\circ}$, the value intensively appearing in the one of the sample of the MF MSs (Fig. 2a) and disappearing after it was calibrated at $800{ }^{\circ} \mathrm{C}$ (Fig. 2c). Similar additional characteristic of the MF-Al MSs is evident from their IR spectrum shown in Fig. $3 \mathrm{~b}$ accompanied by the contrasts of both the MF MSs and the Al MSs in Fig. 3a and c, respectively. The stretching vibrations of $-\mathrm{OH}$ and $-\mathrm{NH}$ overlapped each other around $3318 \mathrm{~cm}^{-1}$, the deformation peak of - NH appeared at $1548 \mathrm{~cm}^{-1}$ and the peaks appeared over a wavenumber range from 1000 to $1500 \mathrm{~cm}^{-1}$ could be attributed to the $-\mathrm{CH}$ deformation vibrations enhancing with its stretching vibration at $2900 \mathrm{~cm}^{-1}$. All these characteristic peaks were intense in the sample of MF MSs and disappeared after the calibration step at $800{ }^{\circ} \mathrm{C}$ as shown in Fig. 2a and c, where the Al-O stretching vibration around $773 \mathrm{~cm}^{-1}$ thereby demonstrates an almost pure alumina product was obtained. 


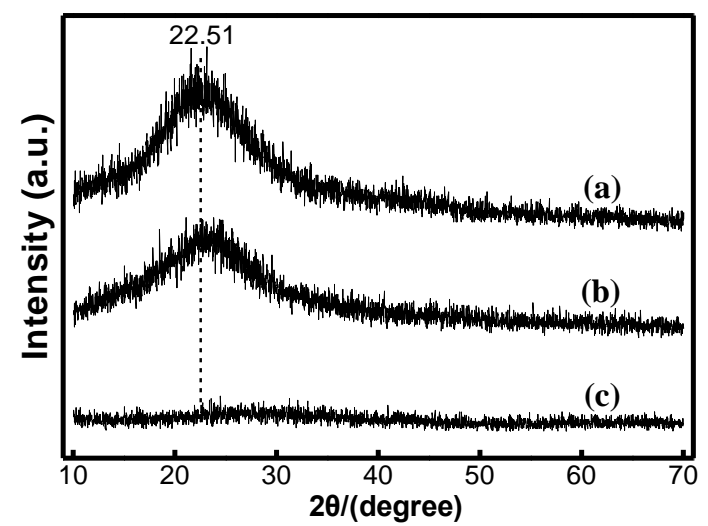

Fig 2 XRD spectra of different MSs

(a) MF MSs; (b) MF-Al MSs; (c) Alumina MSs

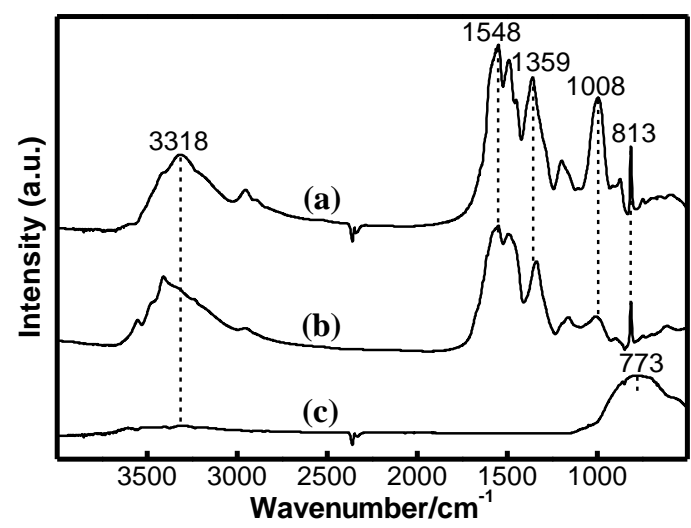

Fig 3 spectra of different MSs

(a) MF MSs; (b) MF-Al MSs; (c) Alumina MSs

The MF and the MF-Al MSs presented an almost entirely consistent diameter $3.2 \mu \mathrm{m}$, the value obtained from their SEM micrographs as shown in Fig. 4 and 5. This consistence in diameter among MF MSs and their hybrids demonstrated it is possible for $\mathrm{Al}^{3+}$ and urea to infiltrate into the MF MSs in the hybridization step. However presence of some fractals in the sample of MF-Al MSs verified it is difficult for a little amount of excessive $\mathrm{Al}(\mathrm{OH})_{3}$ to hybridize with MF-Al MSs and to remain in finally.

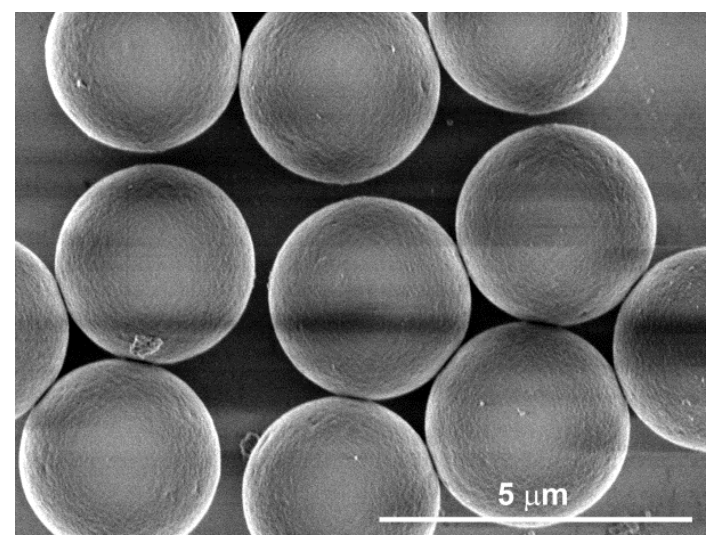

Fig 4 SEM micrographs of MF MSs

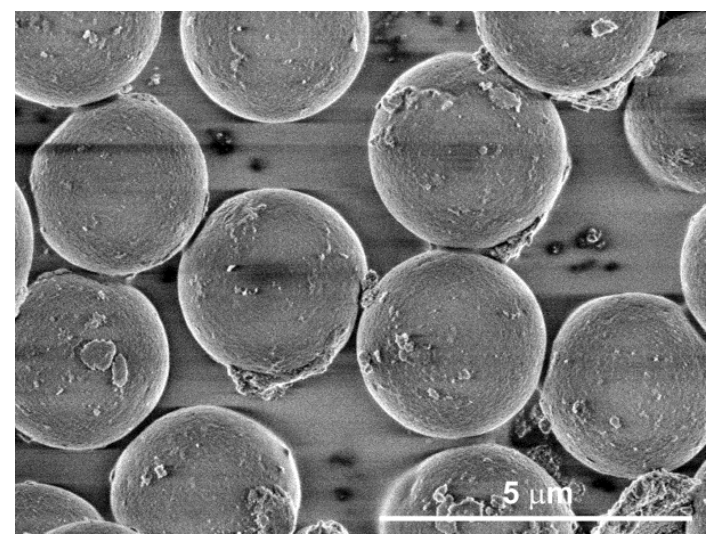

Fig 5 SEM micrographs of MF-Al MSs

The SEM micrographs of the alumina after the hybrids calibrated presented clear profile of MF-Al microspheres, but burst out as shown in Fig. 6. The remained micro-spherical surface as well as the fractal debris from it all demonstrated these MF-Al hybrids indeed have a more $\mathrm{Al}(\mathrm{OH})_{3}$ density on the surface but a more MF resin one in the core. The TG analysis of the MF-Al MSs in Fig. 7 presented a significantly decomposition step spanning a temperature region from 390 to $400{ }^{\circ} \mathrm{C}$ with a weight loss of $22 \%$ and a slowly varying one over a much wide temperature range starting from $400{ }^{\circ} \mathrm{C}$ to $750{ }^{\circ} \mathrm{C}$ with a gradual weight loss over $35 \%$. Obviously dehydration of the MF-Al MSs mainly occurred before $400{ }^{\circ} \mathrm{C}$, and a clear slowly decomposition of organic residue was almost completed after $750{ }^{\circ} \mathrm{C}$. Herein, the overall alumina content c.a. $35 \%$ in the hybrids demonstrated the superior stability of these MF-Al hybrids and these new MF-Al hybrids are expected to be useful in the environment treatment and detection. 

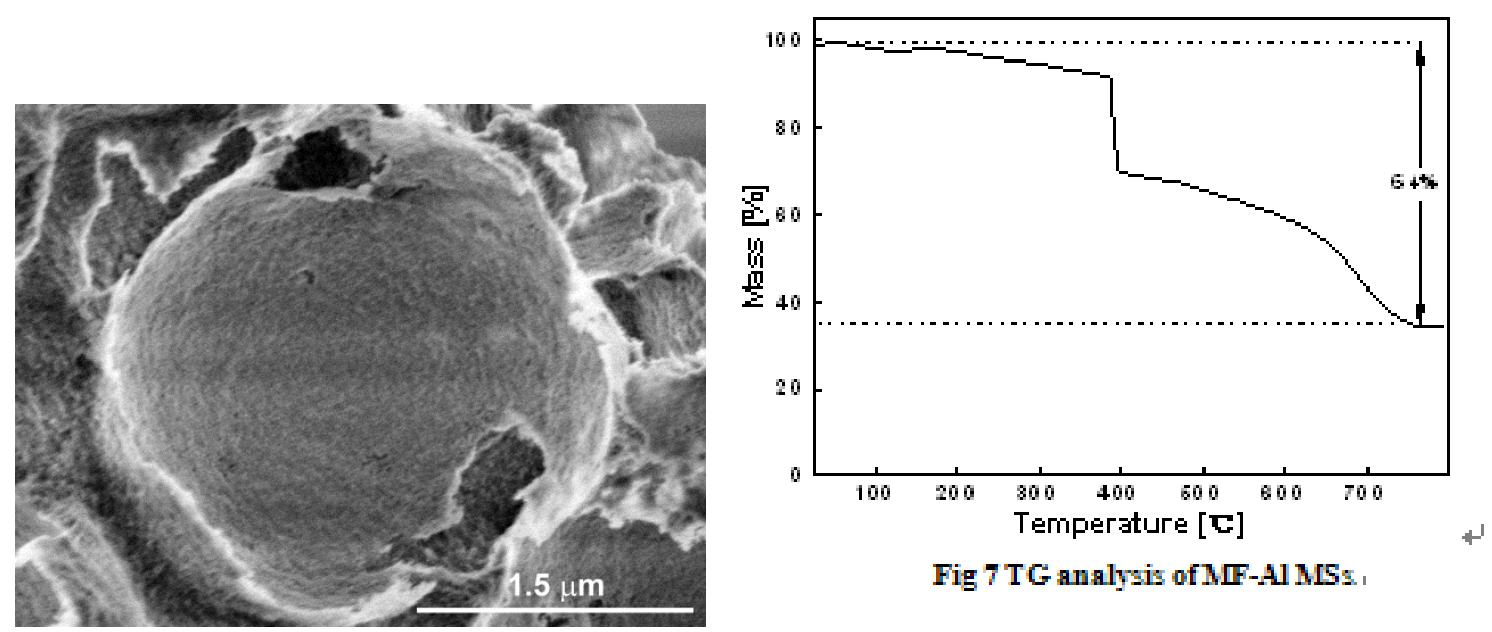

Fig 6 SEM micrographs of alumina MSs

\section{Conclusions}

The melamine formaldehyde resin microspheres hybridized with alumina hydroxide can be simply synthesized by impregnation of melamine formaldehyde microspheres in a reactive solution of aluminum nitrate and urea at $85{ }^{\circ} \mathrm{C}$. The shape of the hybrids can be transcribed from their precursor melamine formaldehyde microspheres. The shape of the melamine-formaldehyde microspheres was further transcribed into the hybrid microspheres, presenting an almost constant diameter $3.2 \mu \mathrm{m}$, the alumina content c.a. $35 \%$, and a superior stability. These characteristics enable it possible for us to design some new applications in environment treatment and detection.

\section{References}

[1] Min, Wang.2002,9(6):44 (in Chinese)

[2] Liu, Y.,Wang, Q. Polymer Degradation and Stability,2006(7):15-17

[3] Z.X. Hao, Z.Li, R.Lin, L.H. Gan, Z.X.Xu, L.W.Chen: Acta Chim. Sinica Vol. 67(2009), p.575(in Chinese)

[4] Zhixian Hao, Yuqing Guo, Mulati Mansuer, Junfu Zhu, Zhirong Zhu Journal of Colloid and Interface Science 430 (2014) 239-248

[5] Jia, G., You, H., Liu, K., Zheng, Y., Guo, N., Zhang, H. Langmuir,2010,26(7):5122-5128

[6] C.du Fresne von Hohenesche, D. F. Schmidt and V. Scha “dler, Chem. Mater., 2008, 20, 6124-6129.

[7] F. Bettina and G. W. Siegmund, Small, 2006, 2, 859-863. 